\title{
The Fracture Energy and Some Mechanical Properties of a Polyurethane Elastomer
}

\author{
H. K. MUELLER* and W. G. KNAUSS**, California Institute of \\ Technology, Pasadena, California 91109
}

\section{Synopsis}

The energy required to form a unit of new surface in the fracture of a polyurethane elastomer is determined. The rate sensitivity of the material has been reduced by swelling it in toluene. This paper primarily describes the experimetal work of measuring the lower limit of the fracture energy. With this value and the creep compliance as a basis, the rate dependence of fracture energy for the unswollen material has been determined. It is thus shown that the dependence of the fracture energy on the rate of crack propagation can be explained by energy dissipation around the tip of the crack. Good agreement between the theoretically and experimentally determined relationships for the rate-sensitive fracture energy is demonstrated.

\section{INTRODUCTION}

In 1953 Rivlin and Thomas ${ }^{1}$ established a criterion for fracture of viscoelastic materials that has the same form as the fracture criterion for elastic materials derived by Griffith ${ }^{2}$ on the basis of energy conservation. Important although not essential ${ }^{3}$ in this derivation is the concept of fracture energy. $\dagger$ Later it was shown by Greensmith and Thomas ${ }^{4}$ that, if this criterion is extended to propagating cracks, the fracture energy becomes a monotonically increasing function of the rate of propagation. Figure 1, which was obtained from the data presented in Ref. 4 for a certain natural rubber vulcanizate, illustrates the rate dependence of the fracture energy.

*Now Research Engineer, Engineering Technology Laboratory, E. I. du Pont de Nemours \& Co. (Ine.), Wilmington, Del. 19898.

**Associate Professor, California Institute of Technology, Pasadena, California. †Sometimes referred to as surface energy. We shall clarify the terminology later.

$$
217
$$

(C) 1971 by The Society of Rheology, Ine.

Published by John Wiley \& Sons, Inc. 


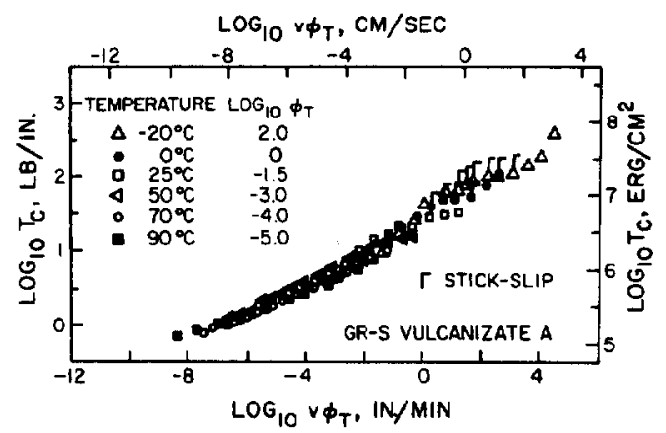

Fig. 1. Shifted tear energy data of Greensmith and Thomas, Ref. 4.

Since the energy dissipation caused by the viscoelasticity of the material is included in the fracture energy, ${ }^{5}$ the question arises as to how much of the rate dependence of the fracture energy is solely attributable to viscous energy dissipation around the crack tip and how much rate sensitivity, if any, remains after this contribution has been subtracted.

In an effort to establish a lower bound on the fracture energy, one is naturally led to investigate the fracture process at temperatures well above the glass-transition temperature and at crack velocities approaching zero. Experimentally this can be difficult, the question being whether the true limit below which propagation is impossible has been reached or merely the limit of the experimentalist's patience. Under normal circumstances, average rates of propagation can be reliably measured down to about $10^{-3} \mathrm{in} . / \mathrm{min}$; cf. Figure 1 . The maximum test temperature is limited by the possibility of thermal degradation of the material. Within the frame of these limitations it may not be possible to observe experimentally the lower bound of fracture energy. The data of Greensmith and Thomas in Figure 1, for instance, show a slight tendency to level off at about $T_{c}=10^{-0.3}$ $\mathrm{lb} / \mathrm{in}$. The experimental data for Solithane, another typical viscoelastic material, exhibit the same general behavior. Nevertheless, it seems speculative to deduce from these data alone that there is a lower bound on $T_{c}$.

Experimental determination of the lower limit of the fracture energy amounts to testing the material under conditions in which the energy dissipation caused by viscosity is negligibly small. It seems natural, 
therefore, to try to remove the internal viscosity sufficiently by swelling the material in a suitable solvent and then performing fracture tests in this state. If the material becomes practically rate insensitive in the swollen state, the fracture criterion for an elastic material may be applied and the fracture energy calculated from experimental data. It is then only necessary to relate this value to its counterpart in fracture of the unswollen material.

The search for the lower limit of fracture energy is of more than academic interest. The existence of a lower limit would guarantee that, under certain long-term loading conditions, crack propagation would not be possible provided chemical changes in the material do not occur. With regard to engineering applications, a lower limit of fracture energy would assure continual service of the structure without eventual failure due to crack propagation.

\section{TERMINOLOGY}

Before we proceed to the experimental determination of fracture energy, it is appropriate to comment on the distinction between fracture and surface energy made in this paper. Depending on one's point of view, the terms fracture energy and surface energy may or may not be interchangeable. On the one hand, surface energy may be defined as the amount of work required to form a unit of new surface, regardless of the molecular processes taking place during the creation of the new surface. On the other hand, some characteristies of the surface-forming process may be introduced into the definition of surface energy. In this sense one may distinguish between work done against intramolecular and intermolecular forces. The latter gives rise to the classical concept of surface tension in liquids. The disruption of chemical bonds would require additional work, which may considerably increase the total work necessary to form a unit of new surface area. In the absence of energy dissipation by other mechanisms, we shall refer to this total work as the intrinsic fracture energy $S$ of the material.

In view of this definition, the energy that must be supplied to form new surface at a given rate may then be called the rate-dependent fracture energy $T_{c}$, or, for short, fracture energy. Inasmuch as a time-dependent stress and strain field around the crack tip causes a certain crack velocity history or vice versa, the fracture energy also 
may somehow depend on this history. We shall demonstrate that for constant crack velocity the fracture energy $T_{c}$, as a function of the magnitude of this constant velocity, can be calculated from a knowledge of intrinsic fracture energy $S$ and creep compliance $D_{\text {er }}$ of the material, assuming that the laws of linear viscoelasticity can be applied to describe the material behavior throughout the strip. The rate-dependent fracture energy hence can be considered as a deduced property instead of a fundamental property of the material.

\section{EXPERIMENTAL DETERMINATION OF INTRINSIC FRACTURE ENERGY $\boldsymbol{S}$}

We shall first describe how the intrinsic fracture energy $S$ can be determined by swelling the material in a suitable agent and performing fracture tests on the material in the swollen state. The result obtained will then be compared to the value calculated from experimental data on the unswollen material at very low crack velocities and temperatures well above the glass-transition temperature. The material chosen for this investigation is a polyurethane elastomer with the tradename Solithane 113. The particular composition used was prepared from equal amounts by volume of the two basic components, resin and catalyst, and will be referred to as Solithane 50/50. The mechanical properties of this material are documented in Ref. 6.

Since $S$ can be determined directly only if the energy dissipation caused by viscosity is negligibly small compared with the magnitude of $S$, we first must show to what degree swelling removes the internal viscosity of the material. The desired effect will be most pronounced if the volume increase by swelling is greatest without severing the integrity of the network. Figure 2 shows the volume increase of Solithane 50/50 in various poorly hydrogen-bonded solvents listed in Table I. The solubility parameter $\delta$ of Solithane $50 / 50$ lies between 9.5 and $10 \sqrt{\mathrm{cal} / \mathrm{cm}^{3}}$. Toluene, with $\delta=8.9 \sqrt{\mathrm{cal} / \mathrm{cm}^{3}}$, was chosen as a suitable swelling agent for our purpose. Although it does not yield the maximum volume increase, it was considered the easiest solvent to work with from the group having solubility parameters near that of Solithane $50 / 50$.

The reduction of internal viscosity by swelling can be gauged by investigating the rate dependence of the stress-strain curve, of 


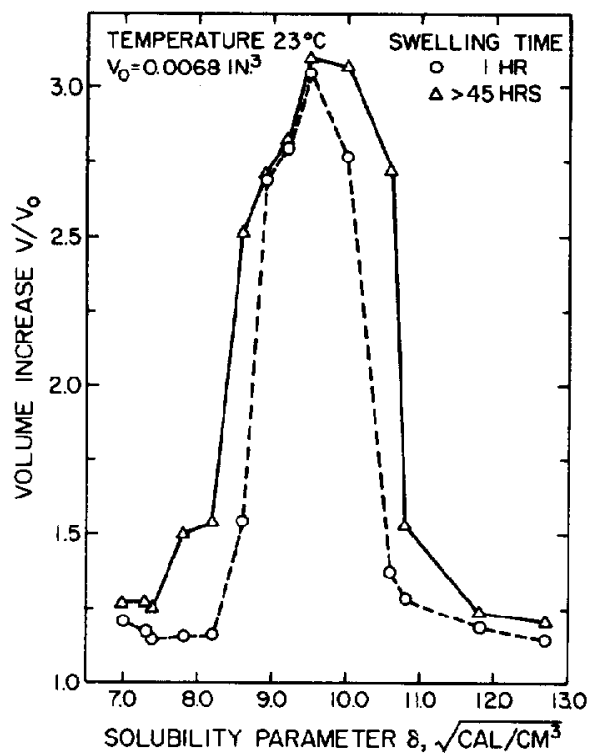

Fig, 2. Swelling ratio of Solithane 50/50 in poorly hydrogen-bonded solvents.

TABLE I

Swelling Agents

\begin{tabular}{|c|c|}
\hline Name & $\begin{array}{c}\text { Solubility Parameter } \\
\text { Ref. } 7 \\
\sqrt{\mathbf{c a l} / \mathbf{c m}^{3}}\end{array}$ \\
\hline n-pentane & 7.0 \\
\hline n-hexane & 7.3 \\
\hline n-heptane & 7.4 \\
\hline methylcyclohexane & 7.8 \\
\hline cyclohexane & 8.2 \\
\hline carbontetrachloride & 8.6 \\
\hline toluene & 8.9 \\
\hline benzene & 9.2 \\
\hline chlorobenzene & 9.5 \\
\hline o-dichlorobenzene & 10.0 \\
\hline l-bromonaphthaline & 10.6 \\
\hline methylcellosolve & 10.8 \\
\hline acetonitrile & 11.8 \\
\hline nitromethane & 12.7 \\
\hline
\end{tabular}


failure data, and of other material responses of the swollen material that exhibit viscoelastic bchavior in the unswollen state. The device pictured in Figure 3 was designed for the tests in the swollen state. It consists of a tank bolted on an Instron crosshead. A spring-loaded clamp is mounted on the tank bottom. Two parallel, vertical rods guide a carriage, which holds the upper clamp and is connected to the Instron load cell. The carriage rides on ball bearings that can be accurately adjusted to achieve parallelism between lower and upper clamps. This feature is important for fracture tests on strip specimens. The displacement of the clamps was monitored by two

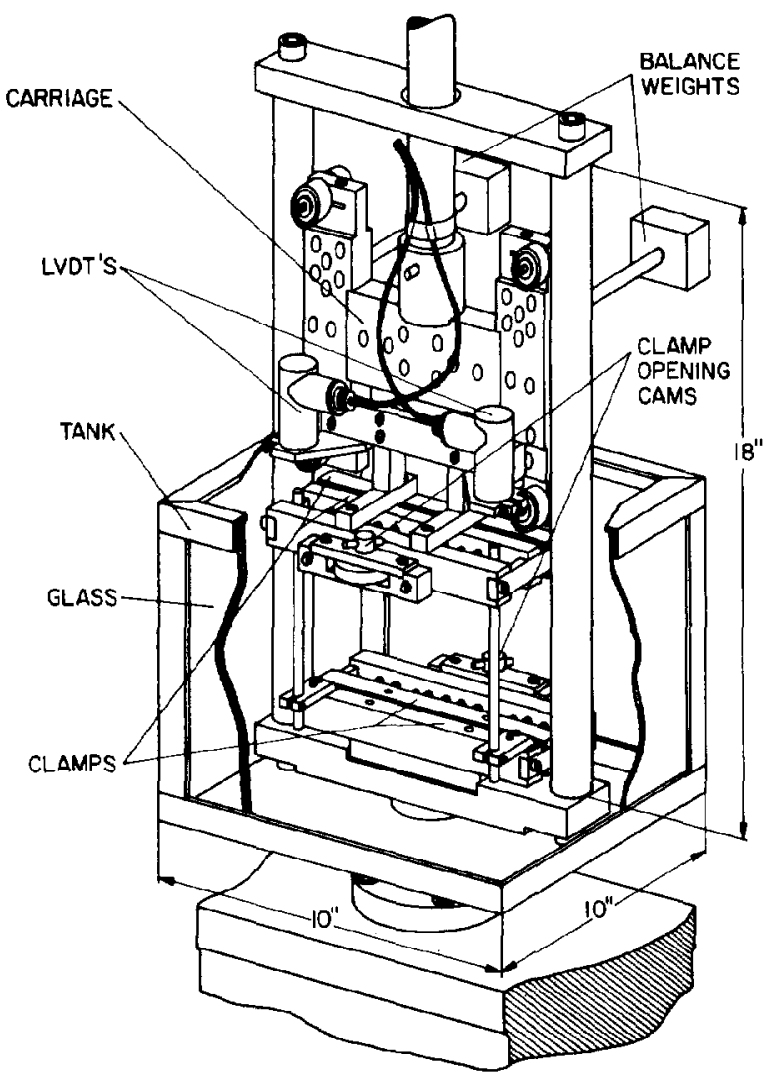

Fig. 3. Device for the mechanical characterization of swollen Solithane. 
linearly variable differential transformers, in addition to the record obtained from the built-in Instron equipment. The clamps can accommodate specimens up to $6 \mathrm{in}$. long and are easily operated by means of clamp-opening cams.

After the specimen had reached its swelling equilibrium, it was inserted in this device and remained submerged throughout the experiment. The test temperature could be adjusted to any level from $-5^{\circ} \mathrm{C}$ to $50^{\circ} \mathrm{C}$ by means of copper coils through which a cooled or heated mixture of ethylene glycol and water was pumped.

Figures 4 and 5 show the uniaxial stress-strain response in the form of Mooney-Rivlin plots for unswollen and swollen Solithane $50 / 50$, which has a glass-transition temperature $T_{g}$ of about $-20^{\circ} \mathrm{C}$. The term $\sigma$ denotes the engineering stress, and $\lambda$ stands for the stretch ratio $1 / 1_{0}$. The response of the unswollen material at $-5^{\circ} \mathrm{C}$ is strongly dependent on strain rate. The stress-strain curves for the swollen material at $-2^{\circ} \mathrm{C}$, on the other hand, are practically independent of rate, although the latter has been changed by a factor

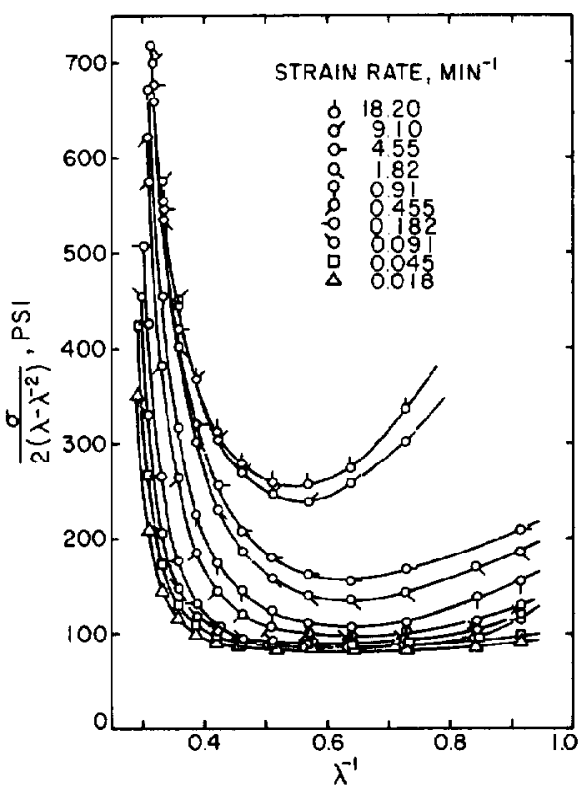

Fig. 4. Mooney-Rivlin plot for unswollen Solithane $50 / 50$ at $-5^{\circ} \mathrm{C}$. 


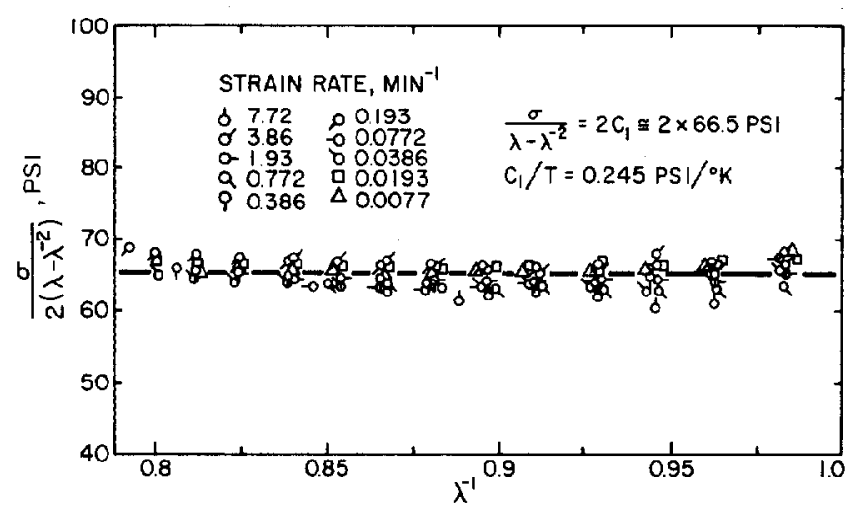

Fig. 5. Mooney-Rivlin plot for Solithane $50 / 50$ swollen in toluene at $-2{ }^{\circ} \mathrm{C}$.

of 1000 . This result indicates a substantially reduced internal viscosity in the swollen material as compared with the unswollen material. The response in the swollen state, furthermore, is seen to be essentially neo-Hookean. Similar tests at higher temperatures also showed that the direct relationship between absolute temperature and the constant $C_{1}$ in the neo-Hookean constitutive law, which is predicted by the classical theory of rubber elasticity,${ }^{8}$ is observed in the swollen state within the limits of experimental error.

The effect of swelling becomes even more obvious when the failure properties, as exemplified by strain at rupture under uniaxial tension, are compared. Figure 6, which gives the strain at failure for unswollen Solithane 50/50, illustrates a strong dependence on strain rate or, via the time-temperature shift principle, on temperature. The strain at failure is seen to vary from about $25 \%$ to roughly $200 \%$, depending on strain rate and temperature, whereas the failure strain of the swollen material exhibits hardly more than a statistical scatter about a certain value for all tested strain rates. The mean value seems to depend slightly on the test temperature, but the data cannot be shifted as in the case of the unswollen material; see Figure 7.

Taking the examples just presented as sufficient evidence that the internal viscosity of the material is reduced to an acceptable level in the temperature range of interest, we may proceed with fracture tests in the swollen state. These tests were carried out on specimens such 


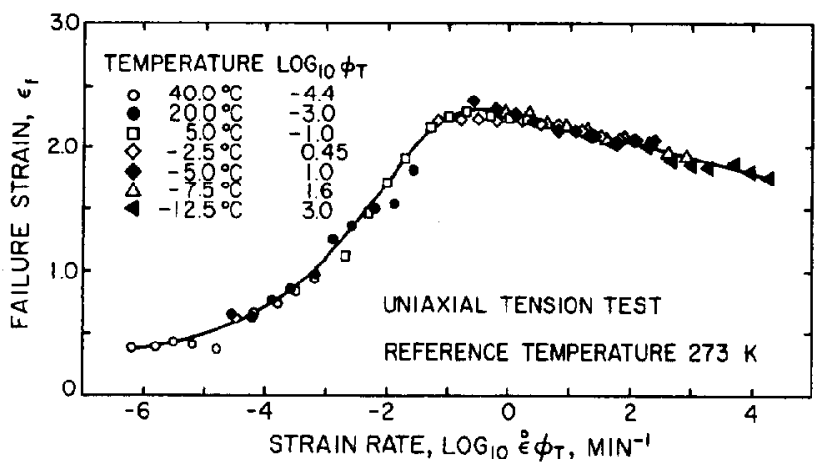

Fig. 6. Failure strains vs. reduced strain rate of unswollen Solithane 50/50.

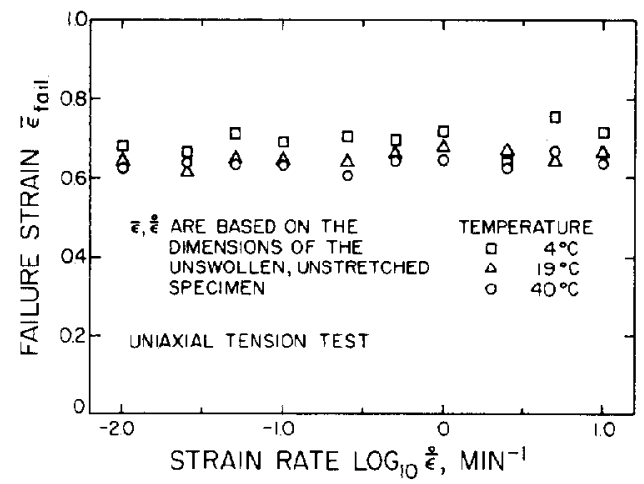

Fig. 7. Failure strain vs. strain rate of Solithane 50/50 swollen in toluene.

as shown in the insert of Figure 8. The specimens were cut from sheets of cast Solithane 50/50 with a thickness of $1 / 32$ in. in the unswollen state. An initial crack of the same length as the strip width was cut in the specimen center on one of the narrow edges. The long edges were clamped in the device already described and displaced at a constant rate. The crack becomes unstable at a certain strain and rapidly propagates through the sheet at a velocity several orders of magnitude higher than in the unswollen state. ${ }^{6}$

Assuming the strip material obeys Hook's law and the stresses around the crack tip to be equal to the ones in an infinitely long 


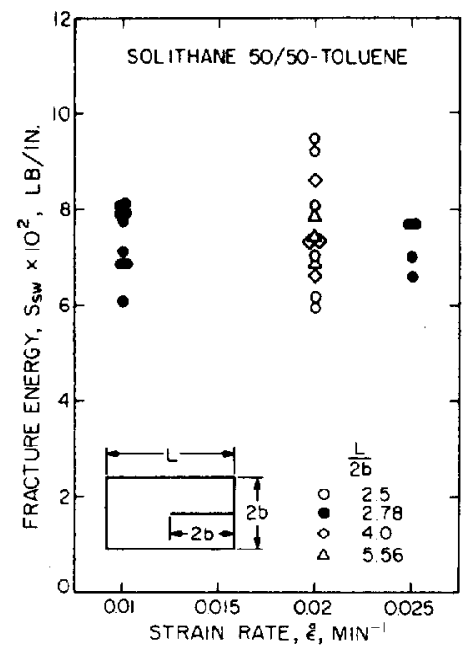

Fig. 8. Fracture energy of swollen Solithane $50 / 50$ at $23^{\circ} \mathrm{C}$.

strip, we can derive the following instability criterion from the stressstrain analysis of this geomotry ${ }^{9} 10$ and for planc stress conditions:

$$
\epsilon_{\mathrm{crit}}=\sqrt{\frac{2 S_{\mathrm{sw}}\left(1-\nu^{2}\right)}{E_{\mathrm{aw}} b}}
$$

where $\epsilon_{\text {orit }}$ is the critical strain at which the crack begins to propagate; $S_{\mathrm{gw}}$ is the intrinsic fracture energy of the swollen material; $E_{\mathrm{gw}}$ is Young's modulus of the swollen material; $b$ is the half strip width, and $v$ is Poisson's ratio of the swollen material.

Only small strains $\epsilon_{\mathrm{crit}}(\sim 2 \%)$ are necessary to cause instability, and the neo-Hookean material behavior is well approximated by Hook's law at strains of this magnitude. As always in these problems, there is some doubt about the material behavior at the crack tip, but we assume the laws of linear elasticity to hold in this region too. The stress analysis of a cracked, infinitely long strip shows that a sufficiently slender strip with a crack of about the same length as its width may be considered as an infinitely long strip with a semiinfinite crack. ${ }^{9,10}$ Tests were run with strips of different aspect ratios to ensure that the finite length of the strip had no effect on the end result. 
Equation (1) furnishes a relationship through which the intrinsic fracture energy of the swollen material can be calculated from the strain at which instability occurs. Young's modulus was determined for each sheet by measuring the slope of the stress-strain curve at zero. Poisson's ratio is taken to be equal to 0.5 . This assumption is not as unreasonable for a swollen material as it might seem. According to the theory of rubber elasticity, ${ }^{8}$ the material can absorb more liquid when it is stretched. The time necessary to complete the diffusion process by which the additional liquid enters, however, is much longer than the duration of any of the fracture tests that were performed $;^{5,11}$ thus the volume increase during the tests could be neglected.

The results of these tests are shown in Figures 8 and 9. The fracture energy of the swollen material is plotted as a function of strain rate, aspect ratio of the strip, and temperature. It should be remembered that, in the swollen state, a distinction between intrinsic fracture energy and rate-dependent fracture energy is not necessary because the material behaves in a brittle manner. Neither the strain rate nor the temperature seems to systematically affect the magnitude of $S_{\mathrm{sw}}$ in the range tested. The data points are scattered over a band, ranging from about $0.06 \mathrm{lb} /$ in. to $0.1 \mathrm{lb} /$ in., which is narrow com-

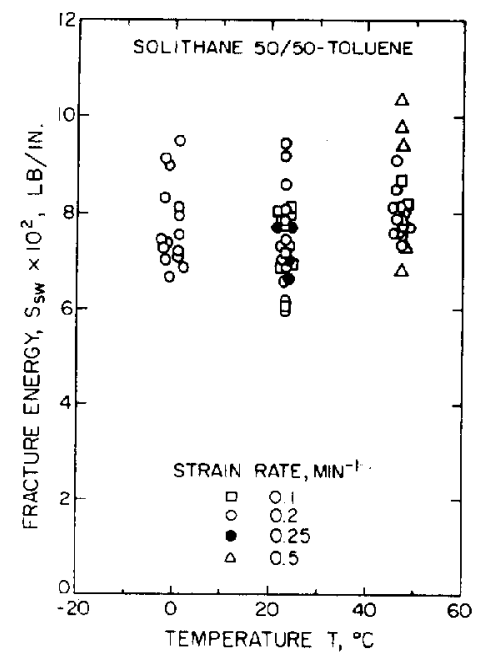

Fig. 9. Fracture energy of swollen Solithane $50 / 50$ as a function of temperature. 
pared with the large range of $S$ obtained for the unswollen material at various crack velocities; ef. Figures 1 and 10 . A certain amount of data scatter has to be expected because the condition of the initial crack tip is important for the onset of fracture. The initial crack was cut with a razor blade, and the shape of the crack tip could not be further controlled. Equation (1) from which $S_{\mathrm{sw}}$ has been calculated is based on the stress field for a line crack having a sharper initial tip than the actual crack tip. The actual stresses at the crack tip will hence be slightly smaller than in theory, and the values of $S_{\mathrm{sw}}$ calculated from eq. (1) will be slightly higher, depending on how much the actual shape of the tip differs from the one of a line crack. The lower limit of $S_{\mathrm{sw}}=0.06 \mathrm{lb} / \mathrm{in}$. will thus be the value that should come closest to the true value.

Having determined the fracture energy in the swollen material, we must now estimate the effect of swelling on this quantity to derive the value of the fracture energy in the unswollen material. We shall do this by assuming with Lake and Thomas ${ }^{2}$ that the intrinsic fracture energy is essentially a measure of the chain-bond strength only.

Consider a sheet of unit thickness to be submerged in a solvent. After swelling equilibrium has been reached, the sheet thickness is $R$. We now extend a crack in the sheet by a small distance $e$. The work necessary to form the new sufrace is

$$
e R S_{\mathrm{sw}}=n_{s} E_{b} e R+2 S_{s}(e / R)
$$

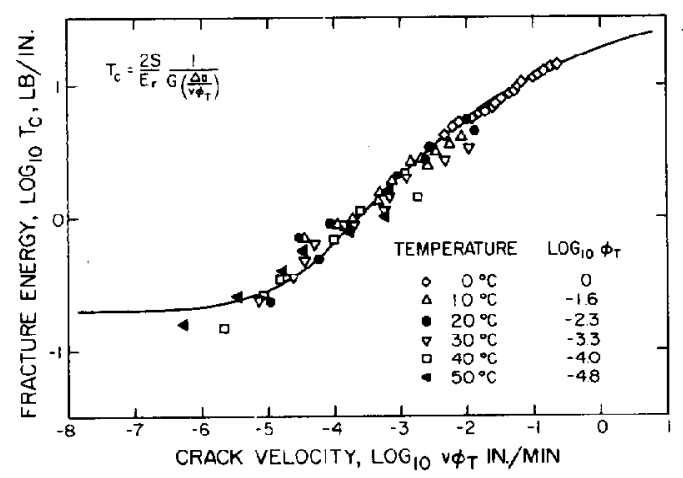

Fig. 10. Fracture energy of Solithane $50 / 50$ as a function of crack velocity and temperature. 
where $n_{s}$ is the number of polymer chains crossing a plane of size unity in the swollen material; $E_{b}$ is the energy required to break a polymer chain, assumed to be unaffected by the presence of the swelling agent, and $S_{s}$ is the surface tension of the interface between swelling agent and polymer.

The last term in eq. (2) accounts for the increase in surface area of the liquid when the chains are broken. The material is assumed to be completely without voids in the unswollen state.

The number of polymer chains $n$ passing through a unit area of unswollen material and the corresponding quantity for the swollen material $n_{s}$ are simply related by

$$
n=n_{s} R^{2}
$$

According to our assumptions, the intrinsic fracture energy of the unswollen material is equal to

$$
S=n E_{b}=n_{s} E_{b} R^{2}
$$

and the following relationship can be obtained from eq. (2)

$$
S=R^{2} S_{\mathrm{sw}}-2 S_{s}
$$

The surface tension of the swelling agent may be assumed to be small (e.g., $S=1.625 \times 10^{-4} \mathrm{lb} /$ in. for toluene in air at $20^{\circ} \mathrm{C}$ ) compared with the quantity $R^{2} S_{\mathrm{sw}}$ and we have approximalely

$$
S=R^{2} S_{w}
$$

For the Solithane 50/50-toluene system a linear swelling ratio $R=$ 1.39 has been found, and with the lower limit of $S_{\mathrm{sw}}$ one calculates a value of $S=0.116 \mathrm{lb} / \mathrm{in}$. for the unswollen material.*

The results of fracture tests with unswollen Solithane 50/50 are presented in Figure 10. A long, narrow strip was used again and the velocity of a crack propagating along the centerline measured as a function of strain.11 From these data the rate-dependent fracture energy $T_{c}$ can be calculated by considering the strain-energy release as the crack proceeds with constant velocity. ${ }^{4}$ In the case of a strip, we define $T_{c}$ by

$$
T_{c}=\frac{E_{\varepsilon_{0} \epsilon_{0}^{2} b}}{1-\nu^{2}} \frac{T}{273}
$$

${ }^{*}$ In Ref. 11 this value was given erroneously as $S=0.0321 \mathrm{lb} / \mathrm{in}$. 
where $E_{\mathrm{r}}$ is the rubbery or long time modulus at $T=273 \mathrm{~K}$; $\epsilon_{0}$ is the lateral strain, and $T$ is absolute temperature.

We obtain the data points in Figure 10 through application of eq. (7), assuming the material to be incompressible. They are plotted on a reduced time scale. A comparison of the time-temperature shift factors listed in this figure with the ones of Figure 6 shows fairly good agreement in temperature dependence.

Taking the value of $T_{c}$ for the two lowest reduced velocities in Figure 10 as a measure of the intrinsic surface energy of Solithane $50 / 50$, one obtains

$$
S=\lim _{v \rightarrow 0} \frac{1}{2} T_{c}(v)=0.08 \mathrm{lb} / \mathrm{in} .
$$

where $v$ denotes the crack velocity. This value is slightly smaller than the one obtained from tests on the swollen material. Taking roughly the average of the values obtained by the two methods, one arrives at the value

$$
S=0.1 \mathrm{lb} / \mathrm{in} .^{2} \pm 20 \%
$$

for the intrinsic fracture energy of Solithane 50/50.

Lake and Thomas ${ }^{11}$ calculated a theoretical value

$$
S=2.0 \times 10^{4} \mathrm{erg} / \mathrm{cm}^{2}=0.114 \mathrm{lb} / \mathrm{in} .
$$

for vulcanized rubber. The network characteristics from which this value is calculated are, to the author's knowledge, unavailable for Solithane 50/50. But the order of magnitude of $S$ may be assumed to be the same for both materials. The agreement between the above values is possibly coincidental but seemed worth mentioning.

For the system considered here, the advantage of determining the intrinsic fracture energy $S$ from tests in the swollen state is easily recognized when the large range of $T_{c}$ is considered in Figure 10. Depending on temperature and crack velocity, the fracture energy may assume any value between roughly 0.16 and $16 \mathrm{lb} / \mathrm{in}$. A whole series of tests must be run to determine the fracture energy-crack velocity relationship to be sure that $S$ is calculated from data at the lower end of this curve. In the case of Solithane 50/50, however, a test in the swollen state would yield a value for $S$ that would differ by a factor of 3 at most from the correct value whereas only one test in the unswollen state may lead to a result which is off by an order of magnitude. 


\section{THE SIGNIFICANCE OF $\boldsymbol{S}$ IN VISCOELASTIC FRACTURE}

We shall now consider the role of the intrinsic fracture energy $S$ in the fracture of viscoelastic materials, in particular how it is related to the rate-dependent fracture energy $T_{c}$; cf. Figures 1 and 10 . The theoretical development of this connection has been presented elsewhere, ${ }^{13}$ and we shall quote only the result necessary for the subsequent analysis and interpretation of experimental data. In the case of the strip geometry already discussed, Ref. 13 asserts that, for a linearly viscoelastic material, the following relationship between lateral strain $\epsilon$, crack velocity $v$, and absolute temperature $T$ holds

$$
\frac{2 S}{E_{r}} \frac{1}{G\left(\Delta a / v \phi_{T}\right)}=\frac{\epsilon_{0}^{2} E_{r} b}{\left(1-\nu^{2}\right)} \frac{T}{273}
$$

where

$$
\begin{aligned}
& G(t)=2\left[\frac{1}{t} \int_{0}^{t} D_{\mathrm{cr}}(\tau) d \tau-\frac{1}{t^{2}} \int_{0}^{t} \tau D_{\mathrm{cr}}(\tau) d \tau\right] \\
& D_{\mathrm{cr}}(t)=\text { creep compliance, } \\
& \Delta a=\text { a length experimentally determined by fitting data, } \\
& \quad \text { about } 150 \AA \text { for Solithane } 50 / 50, \\
& \phi_{T}=\text { time-temperature shift factor. }
\end{aligned}
$$

Comparing eqs. (7) and (11) leads immediately to the expression

$$
T_{c}=\frac{2 S}{E_{r}} \frac{1}{G\left(\Delta a / v \phi_{T}\right)}
$$

which gives the fracture energy as a function of crack velocity and temperature in terms of a rate independent intrinsic fracture energy and a function $G$ of the creep compliance of the material.

It is a simple matter to show that $G(\infty)=D_{\text {cr }}(\infty)$, and it follows that

$$
T_{c}(o)=2 S
$$

The intrinsic fracture energy $S$ is thus equal to half the energy required for rupture if there occurs no energy dissipation through viscous forces around the tip of the advancing crack. It appears reasonable, therefore, to assume that, if the crack velocity varies, the energy that must be supplied for the fracture process depends on the crack velocity because the amount of dissipation around the crack tip depends on this velocity. 
We emphasize that the rate dependence of the fracture energy $T_{c}$ arises solely from energy dissipation due to viscous forces, which can be expressed by other material properties like the creep compliance $D_{\text {cr. }}$. The quantity $T_{c}$ is thus not an intrinsically ratedependent material property arising from the fracture process. To underscore this statement, we compare in Figure 10 the calculated fracture energy $T_{c}$, according to eq. (13), as a function of crack velocity $v$ with the experimentally determined relationship between $T_{c}$ and $v$ for Solithane 50/50. The value of $S$ that has been determined earlier and the function $G(t)$ shown in Figure 11 have been used in the calculation. The reciprocal relaxation modulus $E_{\text {геl }}{ }^{-1}$ and the creep compliance of Solithane 50/50 also are shown in this figure. It can be seen that the main difference between these functions is a shift along the time axis. The fracture energy as a function of crack velocity is easily calculated from the experimental relationship between strain $\epsilon_{0}$ and erack velocity $v$ by considering the change in reversibly stored energy in the strip. ${ }^{1}$ Good agreement between theoretical and experimental dependence of the fracture energy on the crack velocity in Solithane $50 / 50$ is seen to exist over the whole range of velocities.

\section{CONCLUSION}

The fracture energy $T_{c}$ that must be supplied to propagate a crack with a certain velocity through a viscoelastic material is not an intrinsically rate-dependent quantity. It can be calculated from the

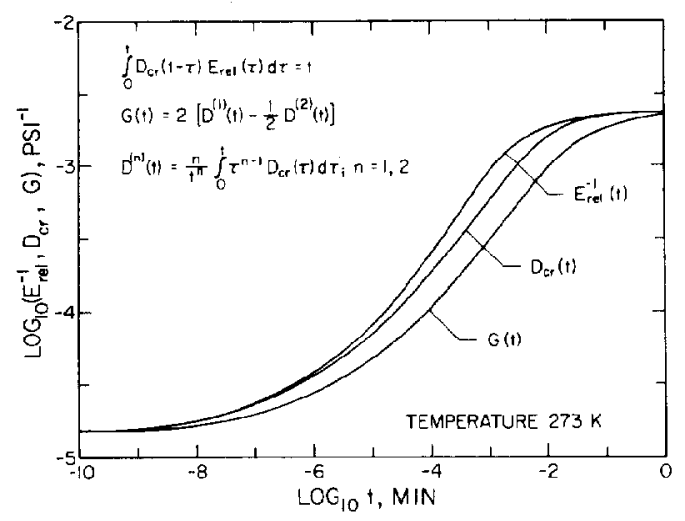

Fig. 11. Relaxation function, creep function and $\mathrm{G}$ function for Solithane 50/50. 
intrinsic fracture energy $S$, which is a rate-independent material property, and the creep compliance $D_{\mathrm{cr}}$ of the material. Good agreement between theory and experiment has been demonstrated for a polyurethane elastomer. The intrinsic fracture energy can be quickly determined from fracture tests of the swollen material.

This work represents part of a Ph.D. thesis submitted to the California Institute of Technology. The authors gratefully acknowledge the sponsorship of the National Aeronautics and Space Administration under Research Grant No. NGL-05-002-005, GALCIT 120, while carrying out this work; and the assistance of the Air Force under the Air Force Rocket Propulsion Laboratory, Research and Technology Division, Edwards, California, Air Force Systems Command, United States Air Force, F04611-67-C-0057, during the preparation of the manuscript.

\section{References}

1. R. S. Rivlin and A. G. Thomas, J. Polymer Sci., X 291 (1953).

2. A. A. Griffith, Phil. Trans. Roy. Soc., 221, 163 (1921).

3. G. I. Barenblatt, "The Mathematical Theory of Equilibrium Cracks in Brittle Fracture," Advan. Appl. Mech., Vol. 7, Academic Press, New York (1962).

4. H. W. Greensmith, and A. G. Thomas, J. Polymer Sci., XVIII, 189 (1955).

5. L. Mullins, Trans. and Proc. Inst. of the Rubber Ind., 35:6, 213 (1959).

6. W. G. Knauss and H. K. Mueller, "The Mechanical Characterization of Solithane 113 in the Swollen and Unswollen State," GALCIT SM 67-8, Cal. Inst. Tech. Pasadena. (1967).

7. Polymer Handbook, J. Brandrup and E. H. Immergut, eds., Interscience Publishers, New York (1966).

8. L. R. G. Treloar, The Physics of Rubber Elasticity, Clarendon Press, Oxford (1958).

9. W. G. Knauss, J. Appl. Mech., 33, 356 (1966).

10. H. K. Mueller, "Stress-Intensity Factor and Crack Opening for a Linearly Viseoclastic Strip with a Slowly Propagating Central Crack," GALCIT SM 69-5, Cal. Inst. Tech. Pasadena (1969); Int. J. Fract. Mech., in press.

11. H. K. Mueller, "Stable Crack Propagation in a Viscoelastic Strip," NASA CR-1279, Washington (1969).

12. G. J. Lake and A. G. Thomas, Proc. Roy. Soc. 300, 108 (1967).

13. H. K. Mueller and W. G. Knauss, "Crack Propagation in a Linearly Viscoelastic Strip," GALCIT SM 68-14, Cal. Inst. Tech. Pasadena (1968); J. Appl. Mech., in press.

Received July 24, 1969

Revised June 18, 1970 